\title{
Novel insights on targeting ferroptosis in cancer therapy
}

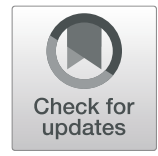

Sipeng Zuo ${ }^{1,2 \dagger}$, Jie $\mathrm{Yu}^{1,2 \dagger}$, Hui Pan ${ }^{1,2^{*}}$ and Linna Lu ${ }^{1,2^{*}}$

\begin{abstract}
Ferroptosis belongs to a novel form of regulated cell death. It is characterized by iron dependence, destruction of intracellular redox balance and non-apoptosis. And cellular structure and molecules level changes also occur abnormally during ferroptosis. It has been proved that ferroptosis exist widespreadly in many diseases, such as heart disease, brain damage or alzheimer disease. At the same time, the role of ferroptosis in cancer cannot be underestimated. More and more indications have told that ferroptosis is becoming a powerful weapon against cancer. In addition, therapies rely on ferroptosis have been applied to the clinic. Therefore, it is necessary to understand this newly discovered form of cell death and its connection with cancer. This review summarizes the mechanism of ferroptosis, ferroptosis inducers based on different targets and inspection methods. At last, we analyzed the relationship between ferroptosis and malignancies, in order to provide a novel theory basis for cancer treatment.
\end{abstract}

Keywords: Ferroptosis, Cancer, Therapy, Signaling pathway

\section{Introduction}

Death is the ultimate destination of cells. The various forms of cell death are apoptosis, necrosis, pyroptosis, oncosis, and autophagy, and each form has its respective features [1]. A disruption of cell death as an approach to treat tumors has been pursued by numerous researchers. As a controlled cellular process, cell death is essential for maintaining tissue integrity and homeostasis, so a disruption of this process under human intervention may show clinical effects [2].

Ferroptosis, another form of cell death, has been observed many times. Cell death caused by insufficient cystine was discovered in the 1950s, and it could be rescued by the lipophilic antioxidant, tocopherol, a component of vitamin E $[3,4]$. Subsequently, lipid peroxidation and reactive oxygen species (ROS) were found to be unfavorable for ferroptosis, and another key enzyme,

\footnotetext{
*Correspondence: xypanhui@163.com; drlulinna@126.com

${ }^{+}$Sipeng Zuo and Jie Yu contributed equally to this work.

'Department of Ophthalmology, Ninth People's Hospital, Shanghai Jiao Tong University School of Medicine, Shanghai, P. R. China

Full list of author information is available at the end of the article
}

glutathione peroxidase 4 (GPX4), was isolated, purified, and confirmed to be involved in the cellular response to oxidative stress [5-7]. Interestingly, GPX4 overexpression has been reported to prevent oxidative stressinduced death in a mouse model [8]. The term ferroptosis, which referred to a novel type of iron-dependent programmed cell death, was introduced in 2012 [9]. Ferroptosis differs from other forms of cell death at biochemical, morphological, and genetic levels. Ferroptosis is characterized by its iron dependence, ability to disrupt the intracellular redox balance, and absence of apoptosis [10]. The differences between ferroptosis and apoptosis lie first in morphological features. When ferroptosis occurs, cell cytoplasm presents round and detached, mitochondrial membrane densities condense, mitochondria crista reduces or vanishes, and outer mitochondrial membrane ruptures [11]. When apoptosis occurs, plasma membrane bubbles, pseudopods retract, and cellular volume reduces, nuclear fragments, chromatin condenses $[11,12]$. In addition, ferroptosis generally is proinflammatory owing to release of damage-associated 
molecular pattern molecules and apoptosis is often antiinflammatory and immune-silent except in some cases with activation of caspases and oligonucleosomal DNA fragmentation. Besides, not the same core regulators play parts in apoptosis and ferroptosis [11]. Studies have demonstrated that ferroptosis is the result of lipid peroxidation, and molecules that directly or indirectly regulate iron metabolism and lipid peroxidation can induce or inhibit ferroptosis. These molecules are mitochondrial voltage-dependent anion channels 2/3 (VDAC2/3), GPX4, ferroptosis suppressor protein 1, heat shock protein (HSP), nuclear factor erythroid 2-related factor 2 (NRF2), nicotinamide adenine dinucleotide phosphate (NADPH) oxidase (NOX), and p53 [11, 13-17]. While some other molecules (p53, Bcl-2 family proteins) are core regulators of apoptosis.

Interestingly, emerging studies have already reported that ferroptosis is related tightly with autophagy [18]. Autophagy refer to a conservative catabolic process that transfer cellular components to lysosomes for degradation [19]. During occurrence of ferroptosis, after cystine deprivation (one of ferroptosis induction condition), which activated autophagy, the iron storage protein ferritin is degraded, which is mediated by the cargo receptor NCOA4. Degraded ferritin results in more Fe releasing, which launch ferroptosis subsequently [18]. In addition, total ROS level of autophagy-deficient cells is significantly lower than autophagy-rescued cells. Therefore, autophagy is required for ROS accumulation in ferroptosis [18]. Because of autophagic specific connection with ferroptosis, drug repositioning therapeutic strategies targeting autophagy to induce cancer cell death are promising [20, 21].

Researchers have reported that ferroptosis can be induced experimentally by various molecules such as the classic inducer, erastin. In addition, ferroptosis has been observed in several types of cancer; therefore, disrupting ferroptosis may offer new treatment options. Here, we discuss the mechanism of action of ferroptosis, describe different ferroptosis inducers according to their targets, and discuss inspection methods. Lastly, the clinical significance of ferroptosis in cancer is presented.

\section{Mechanism of ferroptosis}

System $\mathrm{Xc}^{-}$, a heterodimeric antiporter with important roles in the metabolism of membrane lipids, binds to the twelve-pass transmembrane transporter protein SLC7A11 (xCT) and the single-pass transmembrane regulatory protein SLC3A2 (CD98) via disulfide bonds. Cystine and glutamic acid enter cells through system $\mathrm{Xc}^{-}$on cell membranes. And then the following procedure is the synthesis of glutathione (GSH) as the substrate of GPX4 through $\gamma$-glutamyl cysteine ligase and GSH synthase [22-24]. In general, membrane lipid metabolism is accomplished by the reductase GPX4 and the existence of GSH guarantee GPX4 normal physiological function. A previous study has reported that GPX4, as a key reductase in the pathogenesis of ferroptosis, can mediate the breakdown of membrane lipids [25]. This is the first step involved in ferroptosis to make GPX4 inactivated. Secondly, Iron $(\mathrm{Fe})$ enters cells by binding to the transferrin receptor 1 (TFR1) on cell membranes and then ferrous ions are transported into the cytosol by the divalent metal ion transporter 1. During ferroptosis, membrane lipids bypass the GPX4 pathway instead, they are oxidized into lipid ROS via the Fenton reaction, with electrons provided by ferrous ions, upon some specific induction condition such as cystine deprivation or excess Fe ions. The Fenton reaction is one of the main ways in which ferroptosis occurs [26]. At last, it is the way in which iron and hydrogen peroxide oxidize various substrates and cause cellular damage [27]. Under conditions of low oxygen, lipid ROS attack important intracellular biomolecules, such as DNA, RNA, and proteins, which disrupts the cellular homeostasis and causes the irreversible death of cells $[11,12,28]$, as shown in Fig. 1. And relevant biomarkers are listed in Table 1.

\section{Ferroptosis inducers}

Ferroptosis is characterized by systemXc ${ }^{-}, \mathrm{GPX} 4$, ferrous ions and other biomolecules. Small exogenous molecules, changes in the cellular environment, changes in the activity/function of key biomolecules, abnormal levels of key cellular molecules, and metabolic disturbances can affect ferroptosis. Ferroptosis inducers can be divided into two classes, namely those that target systemXc ${ }^{-}$(class 1) and those that inhibit GPX4 activity (class 2). In addition, ferroptosis can be induced by other inducers (Table 2) and high or low concentrations of intra- or extracellular molecules such as glutamic acid (Glu) or Fe. Clinical studies have reported that classical ferroptosis inducers can inhibit cancer progression and prolong the survival time of patients. Ferroptosis inducers have been used in the synthesis of drug-encased nanoparticles to induce ferroptosis in cancer cells. In the subsequent sections, we summarize the different types of ferroptosis inducers and discuss their mechanisms of action in cancer.

\section{Inhibition of system Xc ${ }^{-}$}

System $\mathrm{Xc}^{-}$has important roles in the transport of amino acids during ferroptosis, suggesting that cancer cells rely on system $\mathrm{Xc}^{-}$. SystemXc ${ }^{-}$inhibitors can inhibit cystine uptake and disrupt protein folding, thereby inducing ferroptosis. 


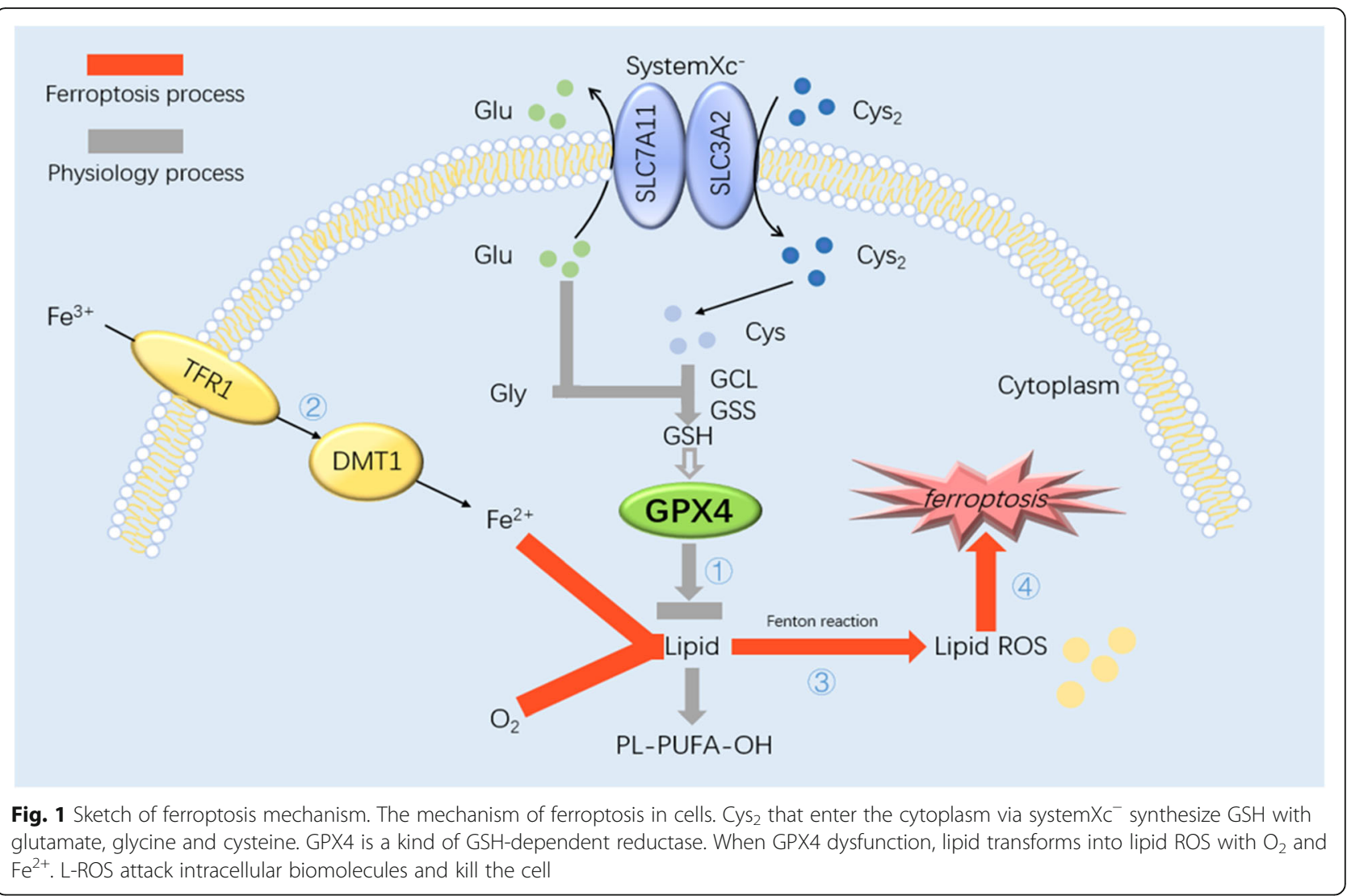

\section{Erastin and derivatives}

Erastin, a small molecule compound discovered by Dolma and colleagues in 2003, selectively kills RAS-expressing cancer cells [29]. Molecular mechanism studies have revealed that mutant $R A S$ can significantly promote the expression of transcription factor NRF2 and further activate the downstream important factor, SLC7A11 [30]. Therefore, erastin is a ferroptosis inducer that down-regulates the GSH level by inhibiting system $\mathrm{Xc}^{-}$, which is caused by the unique structural features of erastin. Erastin also targets VDAC2/3, a mitochondrial membrane exchange channel, and regulates its permeability, causing mitochondrial disorders, production of ROS, and ferroptosis [31, 32]. Furthermore, another study has demonstrated that erastin and its derivatives, such as piperazine erastin, show inducing effectiveness [12]. Additionally, erastin can also affect the breakdown of iron by inhibiting TFR1 [33], and the ferroptosis inhibitor, HSPB1, can reduce the intracellular iron concentration by inhibiting the expression of TFR1. Therefore, HSPB1 overexpression can inhibit ferroptosis [34].

\section{Others}

The effects of Salazosulfapyridine and sorafenib are similar to those of erastin. The United States Food and Drug Administration has approved Salazosulfapyridine for the treatment of rheumatoid arthritis. Similar to erastin,

Table 1 Biomarkers for ferroptosis

\begin{tabular}{lll}
\hline Core molecules markers & Biochemistry & Morphology \\
\hline (1)Pro-ferroptosis: & (1)Activation of MAPKs & (1) Round and detached cell cytoplasm \\
TFR1 & (2)Iron and ROS accumulation & (2)Condensed mitochondrial membrane densities \\
VDAC2/3 & (3)Inhibition of system Xc $C^{-}$with decreased cystine uptake & (3) \\
Ras & (4) GSH depletion & (4) Outer mitochondrial membrane rupture \\
P53 & (5)Increased NAPDH oxidation & \\
NOX & & \\
(2)Anti-ferroptosis: & & \\
SLC7A11 & & \\
HSPB1 & & \\
NRF2 & & \\
GSH & & \\
GPX4 & &
\end{tabular}


Table 2 Ferroptosis inducers

\begin{tabular}{|c|c|c|}
\hline Category & Molecule & Target \\
\hline \multirow[t]{3}{*}{ SystemXc- Inhibition } & Erastin & SystemX $X c^{-}$NDAC/TFR1 \\
\hline & SAS & SystemXc $c^{-}$ \\
\hline & SRF & SystemXc $c^{-}$ \\
\hline \multirow[t]{6}{*}{ GPX4 Inhibition } & $\mathrm{RSL} 3 / 5$ & GPX4/NDAC \\
\hline & FINs & GPX4 \\
\hline & FINO2 & GPX4 \\
\hline & DPIs & GPX4 \\
\hline & Altretamine & GPX4 \\
\hline & Withaferin A & GPX4 \\
\hline \multirow[t]{7}{*}{ Others classes } & GLU & Cystine \\
\hline & Iron carrier & Iron \\
\hline & $\mathrm{BSO}$ & GSH \\
\hline & DPI2 & GSH \\
\hline & Cisplatin & GSH \\
\hline & $\begin{array}{l}\text { Artemisinin and } \\
\text { derivative }\end{array}$ & Iron \\
\hline & Nanoparticle inducers & \\
\hline
\end{tabular}

Salazosulfapyridine can induce ferroptosis by suppressing system $\mathrm{Xc}^{-}$, although the efficacy of Salazosulfapyridine is weaker than that of erastin [35]. Nevertheless, Salazosulfapyridine can enhance the efficacy of other chemotherapeutic drugs that target glioma [36]. By contrast, the concrete mechanism of action of sorafenib remains to be studied, although two approaches have been proposed [37].

\section{GPX4 inhibition and degradation}

System $\mathrm{Xc}^{-}$inhibitors induce ferroptosis by affecting the function of system $\mathrm{Xc}^{-}$. However, some cancer cells can synthesize cysteine and methionine through amino acid metabolic pathways, and systemXc $\mathrm{Xc}^{-}$inhibitors have no effect on these cells. GPX4, as a GSH-dependent enzyme, can suppress the production of lipid ROS. Furthermore, GPX4 is overexpressed in most cancer cells, and the manipulation of GPX4 function can affect the extent of ferroptosis in cancer cells [38].

\section{$R S L 3 / 5$}

RSL refers to RAS Selective Lethal small molecules, which were isolated by the Stockwell laboratory in 2008 [39]. The chloroacetamide moiety of RSL3 is essential for GPX4 function. Specifically, RSL3 combines with GPX4 through the alkylation of the selenocysteine, thereby resulting in the inactivation of GPX4. Furthermore, caspase inhibitors could not reverse RSL3-induced cell death, suggesting that this type of cell death is not caspase-dependent. By contrast, RSL5 requires VDAC2/ 3 , as well as protein synthesis, to induce ferroptosis [39].
FINs

Category II FINS were identified during a large screening of potential ferroptosis inducers. FIN56 (Mr 517.66), a typical FIN derived from CIL56, inhibits the activity of GPX4 without reducing the GSH level. Compared to CIL56, FIN56 is a more potent inducer of ferroptosis $[40,41]$. FIN56 induces ferroptosis through two mechanisms. It can promote the degradation of GPX4 with the aid of acetyl-CoA carboxylase or activate squalene synthase to deplete the antioxidant coenzyme Q10. The oxime moiety in FIN56 is critical for ferroptosis, and the hydrophobicity of the piperidine determines if ferroptosis will be induced $[42,43]$.

\section{Others}

FINO2 is an organic peroxide with a mechanism of action similar to that of artemisinin; however, only the portion of the molecule containing 1,2-dioxolane has been reported to induce ferroptosis. Similar to RSL3, FINO2-induced ferroptosis is not dependent on the caspase pathway; instead, FINO2 deactivates GPX4 and oxidizes Fe directly [37]. Due to the high levels of Fe in cancer cells, ferroptosis is easily induced in cancer cells by FINO2, and its endo-peroxide structure and polar head are largely responsible for this induction. Other small molecules can also inhibit GXP4 through different mechanisms. DPI7 (ML162), DPI10 (ML210), DPI12, DPI13, DPI17-19 inhibit GPX4 by covalent binding, altretamine inhibits GPX4 directly, and withaferin A inactivates GPX4 by increasing the $\mathrm{Fe}^{2+}$ level, and thereby inducing ferroptosis [44].

\section{Other classes}

\section{Glutamic acid}

Ferroptosis has been observed in brains exposed to large amounts of Glu, which may have inhibited the function of system $\mathrm{Xc}^{-}$[45]. Ferroptosis is also involved in ischemia-reperfusion injury in the heart and kidneys under presence of Glu [46, 47].

\section{Iron carriers}

Except for glutamate, superfluous Fe ions can induce ferroptosis, and the modality of iron loading involves various malysites, such as ferrous chloride, ferrous ammonium sulfate, ferric ammonium citrate, and haemin/ hemoglobin. Superfluous Fe ions have been reported to induce lipid peroxidation, ROS accumulation, and cell death [48-50].

\section{GSH synthesis inhibitors}

In the absence of GSH, the function of GPX4 is compromised and the generation of ROS is increased by the Fenton reaction. Buthionine sulfoximine is an inhibitor of GSH synthesis. A low GSH level can also hinder 
ferroptosis. Both DPI2 and cisplatin, as inhibitors of GSH synthesis, can induce ferroptosis by depleting GSH from cells [42].

\section{Artemisinin and its derivatives}

Artemisinin, known for its treatment of malaria, can interfere with the redox balance in cells; therefore, the cytotoxic effects of this compound are closely related to those of Fe ions. Pancreatic ductal adenocarcinoma cells rarely undergo apoptosis, but they can be killed by artesunate-induced ferroptosis. In addition, the artemisinin derivative, DHA, is effective against acute myeloid leukemia, as well as head and neck squamous cell carcinoma, where it has been shown to degrade ferritin by autophagy $[12,51]$.

\section{Nanoparticle inducers}

Nanomaterials are believed to induce ferroptosis by participating in diverse biochemical reactions and interfering with the metabolic balance of cells. In 2016, Kim and colleagues first reported that nanoparticles can induce ferroptosis. They induced ferroptosis in cancer cells by structuring $\alpha-\mathrm{MSH}-\mathrm{PEG}-\mathrm{C}^{\prime}$ dots with near-infrared fluorescent ultra-small silica nanoparticles $\left(C^{\prime}\right.$ dots, $\sim 6$ $\mathrm{nm}$ ), polyethylene glycol (pegylated $\mathrm{C}^{\prime}$ dots), and alphamelanocyte stimulating hormone $(\alpha-\mathrm{MSH})$ and reported an increase in the Fe level, an accumulation of ROS, and a decrease in the GSH level $[52,53]$.

After that, nanoparticle inducers facilitate ferroptosis using a mechanism similar to that of previously mentioned molecules. It's been known that the natural omega 3 fatty acid docosahexaenoic acid depletes GSH, inactivates GPX4, and increases ROS levels $[54,55]$. By contrast, SRF@Fe ${ }^{\mathrm{III}} \mathrm{TA}$ (made up of $\mathrm{Fe}^{3+}$ ion, tannic acid and sorafenib), MON-p53 (metal-organic network encapsulated with p53 plasmid), DGU:Fe/Dox (a nanolongan delivery system releasing $\mathrm{Fe}^{3+}$ and doxorubicin), and PEGylated single-atom Fe-containing nanocatalysts can increase intracellular $\mathrm{Fe}^{2+}$ levels [56-59]. Studies are underway to identify more potent nanoparticles with fewer side effects.

\section{Methods of measurement}

Ferroptosis can be observed and measured by transmission electron microscopy, and the results of these morphological and biochemical studies have revealed that ferroptosis can induce diverse cellular changes, indicating that it affects different molecules. A previous study reported that ferroptotic cells are round and detached, with mitochondrial damage (i.e., shrinkage or loss of cristae, fragmentation of outer membrane); however, nuclei remain intact [12].ROS accumulation is the most important predictor of ferroptosis. ROS levels can be measured by fluorescent sensors, such as $2^{\prime}, 7^{\prime}$ - dichlorodihydrofluorescein diacetate and MitoSOX, as well as other probes such as LiperFluo [60] and isoprostane [61]. For instance, MitoSOX is used to measure ROS levels in mitochondria [62].

GPX4 participates in the breakdown of membrane lipids. It also has important roles in the prevention of cell death. GPX4 activity can be measured by various approaches. In the first approach, the NADPH oxidation rate, which correlates with the reduction of tertbutylhydroperoxide by GPX4, is measured. In the second approach, the phosphatidylcholine hydroperoxide level is quantified by liquid chromatography-mass spectrometry or immunoblot analysis. The level of prominin 2, a stress response protein, can also be measured $[63,64]$.

As previously discussed, Fe can promote ferroptosis. However, there is no method to measure the Fe level in viable cells. Furthermore, it is not useful to measure the total Fe concentration because redox reactions do not require Fe atoms; instead, methods that measure the ferrous ion concentration should be used. RhoNox-1, the first activated fluorescent probe, utilizes the ferrous ionmediated deoxygenation of the tertiary amine $\mathrm{N}$-oxide to determine the ferrous ion concentration in real-time. As such, RhoNox-1 has overcome the disadvantages of chelation-based probes in the measurement of ROS levels [65]. Moreover, FRET iron probe 1, an endoperoxide reactivity-based Förster resonance energy transfer probe, can measure the labile Fe pool in viable cells by destroying endoperoxide bridges in the $\mathrm{Cy} 3$ triplet molecule [66]. By contrast, the binding of chelation-based probes, such as Phen Green and PRA, to substrates is weaker and less selective in the measurement of Fe levels in cells.

\section{Ferroptosis and carcinoma}

Research on the significance of ferroptosis in cancer has recently gained momentum. Cancer cells are more susceptible to ferroptosis due to high cellular activity (Table 3). For instance, the HSP level is elevated in cancer cells, and HSP can inhibit ferroptosis in these cells [85], suggesting that ferroptosis may offer new treatment options. Recent main advancements in the field are summarized in Fig. 2.

\section{Ferroptosis and lymphoma}

Diffuse large B cell lymphoma (DLBCL) is the most common type of non-Hodgkin's lymphoma, accounting for approximately $30-40 \%$ of all cases [86]. DLBCL is sensitive to GPX4-induced ferroptosis [42], and GPX4 expression is negatively correlated with the prognosis. Thus, GPX4 is a promising prognostic predictor [87]. As for Burkitt's lymphoma, Wang and colleagues confirmed that artesunate can activate the ATF4-CHOP-CHAC1 pathway, thereby inhibiting cancer cell proliferation and 
Table 3 Known genes effects on ferroptosis in various cancers

\begin{tabular}{|c|c|c|c|c|}
\hline Tumor type & Gene & Effect & References & Mechanism \\
\hline \multirow[t]{2}{*}{ Lymphoma } & GPX4 & Inhibition & Yang, W.S et al. [35] & Reduce the accumulation of ROS \\
\hline & CHACl & Promotion & Wang et al. [65] & Degrade GSH \\
\hline \multirow[t]{5}{*}{$\mathrm{HCC}$} & NRF2 & Inhibition & Sun, $X$ et al. [67] & Encode antioxidant proteins \\
\hline & $\mathrm{HICl}$ & Promotion & Xiao Zhang et al. [68] & Affect the synthesis of GSH \\
\hline & HNF4A & Inhibition & & \\
\hline & CISD1 & Inhibition & Yuan, $\mathrm{H}$ et al. [69] & Suppress mitochondrial lipid peroxidation \\
\hline & GABPB1-AS1 & Promotion & Qi, W et al. [70] & Inhibited the cellular antioxidant capacity \\
\hline \multirow[t]{3}{*}{ RCC } & GPX4 & Inhibition & Yang et al. [35] & Reduce the accumulation of ROS \\
\hline & TAZ/NOX & Promotion & Yang et al. [71] & Exhaust intracellular NADPH \\
\hline & BAP1 & Inhibition & Zhang, Y et al. [14] & Decreases SLC7A11 expression \\
\hline \multirow[t]{2}{*}{ Ovarian cancer } & TFR1 & Promotion & Pizzamiglio et al. [72] & Increase the intake of iron \\
\hline & TAZ/NOX & Inhibition & Yang et al. [73] & Reduce the depletion of NADPH \\
\hline \multirow[t]{3}{*}{ Pancreatic cancer } & HSPA5 & Inhibition & Zhu, S et al. [74] & Inhibit the expression of TFR1 \\
\hline & System Xc & Inhibition & Lo M et al. [75] & Increase the synthesis of GSH \\
\hline & GRP78 & Inhibition & Wang, K et al. [76] & Unkown \\
\hline \multirow[t]{4}{*}{ Breast cancer } & TFR1 & Promotion & Basuli et al. [77] & Increase the intake of iron \\
\hline & CHACl & Promotion & Chen et al. [78] & Degrade GSH \\
\hline & ACSL4 & Promotion & Doll, S et al. [79] & Increasing cellular lipid composition \\
\hline & P53RRA & Promotion & Chao Mao et al. [80] & Nuclear sequestration of p53 \\
\hline Head and neck cancer & NRF2 & Inhibition & Sun, $\mathrm{X}$ et al. [81] & Encode antioxidant proteins \\
\hline Colorectal cancer & P53 & Inhibition & Xie Y et al. [82] & Increase the expression of system X $\mathrm{C}^{-}$ \\
\hline $\begin{array}{l}\text { Cervical carcinoma } \\
\text { Melanoma }\end{array}$ & $\begin{array}{l}\text { HSPB1 } \\
\text { SLCIA5 } \\
\text { GOT1 }\end{array}$ & $\begin{array}{l}\text { Inhibition } \\
\text { Promotion } \\
\text { Inhibition }\end{array}$ & $\begin{array}{l}\text { Stuart et al. [62] Sun, X et al. [29] } \\
\text { Luo, M et al. [83] } \\
\text { Zhang, K et al. [84] }\end{array}$ & $\begin{array}{l}\text { Inhibit the expression of TFR1 } \\
\text { Reduce the accumulation of Glu } \\
\text { Decrease the depletion of Glu }\end{array}$ \\
\hline
\end{tabular}

inducing ferroptosis [67]. In addition, $A L O X 12$ can promote ferroptosis in $p 53$-dependent cancers without affecting GPX4 function [88]. Deletion of the ALOX12 allele can accelerate tumorigenesis in an $\mathrm{E} \mu$-myc lymphoma model, suggesting that it may be a potential therapeutic target for $p 53$-dependent tumors.

\section{Ferroptosis and hepatocellular cancer}

Hepatocellular carcinoma is often diagnosed at late stages, and it is ranked as the third cause of mortality in males worldwide [89]. Sorafenib, a non-specific kinase inhibitor, is used in the treatment of advanced hepatocellular carcinoma, prolonging the survival of patients by several months. Sorafenib can induce ferroptosis in hepatic cancer cells independent of its kinase inhibitory effect $[24,90]$, although drug resistance is a common manifestation of treatment. Drug resistance is caused by the activation of the p62-Keap1-NRF2 pathway. The degradation of NRF2 is inhibited by the binding of p62 to Keap1, which leads to the accumulation of NRF2 in cells. NRF2 can up-regulate diverse ferroptosis inhibitors, such as NQO1 and FTH1, and sensitize hepatic cancer cells to sorafenib and erastin both in vitro and in vivo. Furthermore, high NRF2 levels in cells can promote the transcription of genes that encode antioxidant proteins, thereby protecting hepatic cancer cells from ferroptosis [68].

Retinoblastoma protein $(\mathrm{Rb})$ is an important regulator of cell proliferation [69], and gene mutations can promote hepatocellular carcinoma. Ferroptosis has been observed in sorafenib-treated hepatic cancer cells with negative $\mathrm{Rb}$ expression, with a two- to three-fold increase in cell death [70]. Moreover, the resistance of hepatic cancer cells to sorafenib has also been reported to involve metallothionein-1G [91] and sigma 1 receptor [92]. Zhang and colleagues studied two transcription factors, namely HIC1 and HNF4A, and reported that decreased HIC1 expression promotes ferroptosis, whereas increased HNF4A expression inhibits ferroptosis [71]. In addition, CDGSH iron sulfur domain 1 overexpression in human hepatic cancer cell lines, such as HepG2 and Hep3B, can inhibit mitochondrial lipid peroxidation and ferroptosis [93].

Long non-coding RNAs (LncRNAs) have important roles in ferroptosis. For instance, lncRNA GABPB1-AS1 can down-regulate the GABPB1 level and inhibit its 


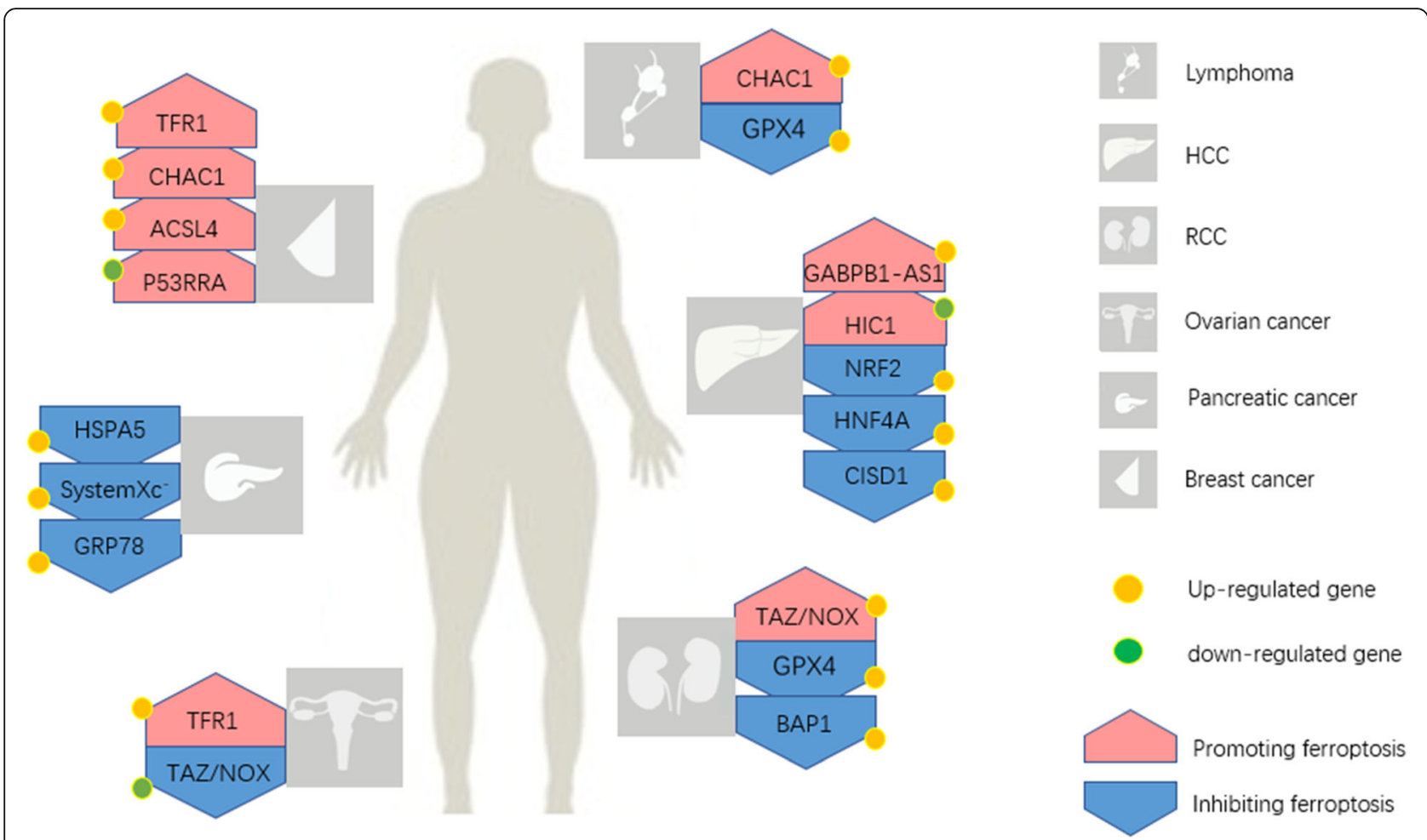

Fig. 2 Known genetic effects on various cancers via ferroptosis. The known genetic effects via ferroptosis in dominating cancers. These cancers include lymphoma, HCC, RCC, ovarian cancer, pancreatic cancer, and breast cancer. They have different sensitivities to ferroptosis

antioxidant capacity, thereby inducing ferroptosis in hepatic cancer cells [77].

\section{Ferroptosis and renal cell cancer}

Clear cell renal cell carcinoma (CCRCC) is the most prevalent type of kidney cancer. CCRCC cells lacking GPX4 expression are susceptible to death because of ROS accumulation [94]. Hereditary leiomyomatosis and renal cell cancer is a rare disease caused by the inactivation of fumarate hydratase in the Krebs cycle, which results in the accumulation of fumarates and the alteration of GPX4 function, thereby increasing the sensitivity of cancer cells to ferroptosis [73].

RCC cells express density-dependent TAZ, an effector in the Hippo-YAP/TAZ pathway, which are generally regarded as oncogene highly conserved in evolution. TAZ can promote the expression of epithelial membrane protein 1 and NOX4, which accelerate the kinetics of ferroptosis by increasing the accumulation of ROS [95]. In addition, it's been found that in renal cancer cell line, as a nuclear deubiquitinating enzyme, the tumour suppressor BRCA1associated protein 1 decreases SLC7A11 expression, thereby causing lipid peroxidation and ferroptosis [16].

\section{Ferroptosis and ovarian cancer}

Similar to hepatocellular carcinoma, ovarian cancer is often diagnosed at late stages [96]. Ferroptosis can enhance the chemosensitivity of ovarian cancer cells. For instance, serous ovarian cancer cells overexpressing TFR1 are sensitive to ferroptosis [97], whereas ovarian cancer tumor-initiating cells and ovarian cancer cells are sensitive to ferroptosis inducers [97]. Erastin can reverse the resistance of ovarian cancer cells to docetaxel [98]. Similar to renal cell carcinoma, the TAZ/NOX pathway mediates ferroptosis and chemoresistance in ovarian cancer cells, and low TAZ expression in patients with recurrent ovarian cancer is responsible for the reduced susceptibility to ferroptosis [74].

Artensunate, as a derivative of artemisinin and an inducer of ferroptosis, can inhibit the proliferation of primary ovarian cancer cells and ovarian cancer cell lines [99]. Meanwhile, the mechanism of action of ferroptosis also involves the enzyme, stearoyl-CoA desaturase 1 (SCD1), which stimulates fatty acid synthesis. If SCD1 is inhibited, then the anti-tumor effects of ferroptosis will be enhanced. Therefore, the combined use of an SCD1 inhibitor and a ferroptosis inducer may represent a new therapeutic approach $[75,100]$.

\section{Ferroptosis and pancreatic cancer}

Pancreatic cancer is a highly lethal disease [101]. HSPA5, a member of the HSP70 family, negatively regulates ferroptosis in human pancreatic ductal adenocarcinoma cells [76]. Furthermore, a previous study reported that 
artensunate can induce ferroptosis in pancreatic ductal adenocarcinoma cells expressing constitutively active KRas, although normal cells were not affected [51]. In addition, system $\mathrm{Xc}^{-}$has important roles in pancreatic cancer and drug resistance because of its function in the transport of cystine, and dysfunction of system $\mathrm{Xc}^{-}$has curative effect on pancreatic cancer [72, 102, 103]. Finally, glucose-regulated protein 78 (GRP78) is a molecular chaperone, and the activation of GRP78 can suppress ferroptosis in pancreatic cancer caused by a KRAS mutation [78].

\section{Ferroptosis and breast cancer}

Breast cancer is one of the most prevalent cancers in women worldwide and has witnessed an improvement in its prognosis due to advancements in the field [79]. The elevated expression of TFR1 in breast cancer cells renders them susceptible to ferroptosis, which may represent a new therapeutic approach [80]. It's reported that siramesine and lapatinib are effective ferroptosis inducers in breast cancer [104]. However, an elevated estrogen level can reduce sulfasalazine-induced ferroptosis through the transferrin receptor [81], suggesting that hormones affect ferroptosis.

$\mathrm{CHAC} 1$ as a downstream protein of activator of transcription factor 4 (ATF4) can degrade GSH [105]. $\mathrm{CHAC} 1$ induces ferroptosis in human triple negative breast cancer cells via the GCN2-eIF2 $\alpha-\mathrm{ATF} 4$ pathway [106]. Except that, acyl-CoA synthetase long-chain family member 4 can promote ferroptosis by increasing the content of intracellular lipids [82]. Another study reported that the down-regulation of lncRNA P53RRA promoted ferroptosis by sequestering p53 in the nucleus [83]. In addition, as a herb widely used in traditional Chinese medicine, danshen has been reported to improve the prognosis of breast cancer, which may be due to the effects of its bioactive compound, dihydroisotanshinone I, in repressing the protein GPX4 expression and inducing ferroptosis [84].

\section{Ferroptosis and other cancer}

Ferroptosis has also been observed in other types of cancers such as prostate and cervical cancer [34]. Head and neck cancer expressing a low level of NRF2 is sensitive to drug-induced ferroptosis. Therefore, the activation of the NRF2-ARE pathway protects cells from the effects of ferroptosis inducers [107, 108]. Mutations in p53 also protect colorectal cancer cells from the effects of erastin-induced ferroptosis [109], which may offer a new treatment option. Moreover, ferroptosis is uncommon in cervical carcinoma cells highly expressing HSPB1 according to recent reports [34, 85], suggesting HSPB1 provide protective effects on cancer cells.
In melanoma, decreased SLC1A5 expression has been reported to reduce glutamine accumulation, decrease Glu production, and increase ferroptosis [110]. In addition, the decrease in glutamic-oxaloacetic transaminase prevented the depletion of Glu, thereby resulting in anti-ferroptosis of melanoma cells [111].

In gastrointestinal cancer, study claimed that CD44v, the variant of CD44 (an adhesion molecule overexpressed in cancer stem-like cells), can interact with the $\mathrm{xCT}$, thereby stabilizing the intracellular level of GSH and defensing against ferroptosis [112]. Given that sulfasalazine (SSZ) inhibits $\mathrm{xCT}$, it is highly likely that SSZ induces ferroptosis of cancer stem-like cells. Therefore, therapeutic strategies that target inducing ferroptosis of cancer stem cells through the molecules localized by $\mathrm{xCT}$ may be pretty promising $[20,113]$.

\section{Conclusions}

Ferroptosis, a novel form of cell death, is characterized by iron dependence, disruption of the intracellular redox balance, and absence of apoptosis. The application of ferroptosis in the treatment of cancer has recently gained momentum, as the process can inhibit tumor growth and improve the efficacy of chemotherapeutic drugs.

Fe, a trace element, is present in high concentrations in the human body. Interestingly, its concentration is higher in cancer cells than that in normal cells. Presently, chemotherapeutic drugs are widely used to kill cancer cells. However, cancer cells can develop resistance to drugs, leading to poor prognosis and low survival rates. Studies have reported an association between ferroptosis and drug resistance. Therefore, developing new chemotherapeutic drugs or eliminating drug resistance by inducing ferroptosis may represent a feasible approach. Studies should also examine the types of cancers that are very sensitive to ferroptosis as well as the genes/ proteins that are up- and down-regulated.

Ferroptosis has been observed in several diseases with many genes involved as above. At this point, it is important to establish the optimal doses of ferroptosis inducers in order to reduce side effects. Ferroptosis can not only cure tumors, but also cause untoward effects in body. Ferroptosis cannot be experimentally induced by simply removing antioxidants from cells; therefore, ferroptosis is by no means a simple Fenton reaction. However, ferroptosis, as an important process, may offer new treatment options as our understanding of the process increases.

\section{Abbreviations}

ROS: Reactive oxygen species; GPX4: Glutathione peroxidase 4; VDAC2/ 3: Voltage-dependent anion channels 2/3; HSP: Heat shock protein; NRF2: Nuclear factor erythroid 2-related factor 2; NADPH: Nicotinamide adenine dinucleotide phosphate; NOX: Nicotinamide adenine dinucleotide 
phosphate oxidase; GSH: Glutathione; TFR: Transferrin receptor 1; Glu: Glutamic acid; DLBCL: Diffuse large B cell lymphoma; Rb: Retinoblastoma protein; LncRNA: Long non-coding RNA; SCD1: Stearoyl-CoA desaturase 1; GRP78: Glucose-regulated protein 78; ATF4: Activator of transcription factor 4

\section{Acknowledgements}

Not applicable.

\section{Authors' contributions}

Sipeng Zuo and Jie Yu wrote and edited the manuscript. Linna Lu and Hui Pan revised this manuscript. All authors approved the final manuscript.

\section{Funding}

This project was supported by National Natural Science Foundation of China (Grant 81500757, 81702781), National Key Research and Development Plan (2018YFC1106100) and The Science and Technology Commission of Shanghai (17DZ2260100, 19JC1410200). The funders played no roles in the study design, decision to publish, or preparation of the manuscript.

\section{Availability of data and materials}

Not applicable.

\section{Ethics approval and consent to participate}

Not applicable.

\section{Consent for publication}

Not applicable.

\section{Competing interests}

The authors declare that they have no competing interests.

\section{Author details}

'Department of Ophthalmology, Ninth People's Hospital, Shanghai Jiao Tong University School of Medicine, Shanghai, P. R. China. ${ }^{2}$ Shanghai Key Laboratory of Orbital Diseases and Ocular Oncology, No. 12, Lane 833, Zhizaoju Road, Huangpu District, Shanghai 200001, P. R. China.

Received: 12 July 2020 Accepted: 21 September 2020

Published online: 02 October 2020

\section{References}

1. D'Arcy MS. Cell death: a review of the major forms of apoptosis, necrosis and autophagy. Cell Biol Int. 2019;43:582-92.

2. Galluzzi $L$, et al. Molecular mechanisms of cell death: recommendations of the nomenclature committee on cell death 2018. Cell Death Differ. 2018;25: 486-541.

3. Eagle $\mathrm{H}$. The specific amino acid requirements of a human carcinoma cell (stain HeLa) in tissue culture. J Exp Med. 1955;102:37-48.

4. Bannai S, Tsukeda H, Okumura H. Effect of antioxidants on cultured human diploid fibroblasts exposed to cystine-free medium. Biochem Biophys Res Commun. 1977;74:1582-8.

5. Ursini F, Maiorino M, Valente M, Ferri L, Gregolin C. Purification from pig liver of a protein which protects liposomes and biomembranes from peroxidative degradation and exhibits glutathione peroxidase activity on phosphatidylcholine hydroperoxides. Biochim Biophys Acta. 1982;710:197211.

6. Yagi K, et al. Expression of human phospholipid hydroperoxide glutathione peroxidase gene for protection of host cells from lipid hydroperoxidemediated injury. Biochem Biophys Res Commun. 1996;219:486-91.

7. Arai M, et al. Mitochondrial phospholipid hydroperoxide glutathione peroxidase plays a major role in preventing oxidative injury to cells. J Biol Chem. 1999;274:4924-33.

8. Ran $Q$, et al. Transgenic mice overexpressing glutathione peroxidase 4 are protected against oxidative stress-induced apoptosis. J Biol Chem. 2004;279: 55137-46.

9. Dixon SJ, et al. Ferroptosis: an iron-dependent form of nonapoptotic cell death. Cell. 2012;149:1060-72

10. Ursini F, Maiorino M. Lipid peroxidation and ferroptosis: the role of GSH and GPx4. Free Radic Biol Med. 2020;152:175-85.

11. Xie Y, et al. Ferroptosis: process and function. Cell Death Differ. 2016;23:36979 .
12. Liang $C$, Zhang $X$, Yang $M$, Dong $X$. Recent Progress in Ferroptosis inducers for Cancer therapy. Adv Mater. 2019;31:e1904197.

13. Tang $Q$, et al. Ferroptosis is newly characterized form of neuronal cell death in response to arsenite exposure. Neurotoxicology. 2018;67:27-36.

14. Bersuker K, et al. The CoQ oxidoreductase FSP1 acts parallel to GPX4 to inhibit ferroptosis. Nature. 2019;575:688-92.

15. Liu Q, Wang K. The induction of ferroptosis by impairing STAT3/Nrf2/GPx4 signaling enhances the sensitivity of osteosarcoma cells to cisplatin. Cell Biol Int. 2019;43:1245-56.

16. Zhang $Y$, et al. BAP1 links metabolic regulation of ferroptosis to tumour suppression. Nat Cell Biol. 2018;20:1181-92.

17. Kang R, Kroemer G, Tang D. The tumor suppressor protein p53 and the ferroptosis network. Free Radic Biol Med. 2019;133:162-8.

18. Gao M, et al. Ferroptosis is an autophagic cell death process. Cell Res. 2016; 26:1021-32

19. Jiang $X$, Overholtzer $M$, Thompson CB. Autophagy in cellular metabolism and cancer. J Clin Invest. 2015:125:47-54.

20. Yoshida GJ. Therapeutic strategies of drug repositioning targeting autophagy to induce cancer cell death: from pathophysiology to treatment. J Hematol Oncol. 2017;10:67

21. Yoshida GJ. The therapeutic strategy of drug re-positioning to induce autophagic cell death in brain malignancy. Apoptosis. 2020;25:457-8.

22. Yang WS, Stockwell BR. Ferroptosis: death by lipid peroxidation. Trends Cell Biol. 2016;26:165-76

23. Imai H, Matsuoka M, Kumagai T, Sakamoto T, Koumura T. Lipid peroxidationdependent cell death regulated by GPx4 and Ferroptosis. Curr Top Microbiol Immunol. 2017;403:143-70.

24. Yu H, Guo P, Xie X, Wang Y, Chen G. Ferroptosis, a new form of cell death, and its relationships with tumourous diseases. J Cell Mol Med. 2017;21:64857.

25. Seibt TM, Proneth B, Conrad M. Role of GPX4 in ferroptosis and its pharmacological implication. Free Radic Biol Med. 2019:133:144-52.

26. Wang S, et al. Iron and magnetic: new research direction of the ferroptosisbased cancer therapy. Am J Cancer Res. 2018:8:1933-46.

27. Winterbourn CC. Toxicity of iron and hydrogen peroxide: the Fenton reaction. Toxicol Lett. 1995:82-83:969-74.

28. Cao JY, Dixon SJ. Mechanisms of ferroptosis. Cell Mol Life Sci. 2016;73:2195209.

29. Dolma S, Lessnick SL, Hahn WC, Stockwell BR. Identification of genotypeselective antitumor agents using synthetic lethal chemical screening in engineered human tumor cells. Cancer Cell. 2003;3:285-96.

30. Yang H, Xiang S, Kazi A, Sebti SM. The GTPase KRAS suppresses the p53 tumor suppressor by activating the NRF2-regulated antioxidant defense system in cancer cells. J Biol Chem. 2020;295:3055-63.

31. DeHart DN, et al. Opening of voltage dependent anion channels promotes reactive oxygen species generation, mitochondrial dysfunction and cell death in cancer cells. Biochem Pharmacol. 2018;148:155-62.

32. Yagoda $\mathrm{N}$, et al. RAS-RAF-MEK-dependent oxidative cell death involving voltage-dependent anion channels. Nature. 2007:447:864-8.

33. Feng $\mathrm{H}$, et al. Transferrin Receptor Is a Specific Ferroptosis Marker. Cell Rep. 2020;30:3411-23.e3417.

34. Sun $X$, et al. HSPB1 as a novel regulator of ferroptotic cancer cell death. Oncogene. 2015:34:5617-25.

35. Dixon SJ, et al. Pharmacological inhibition of cystine-glutamate exchange induces endoplasmic reticulum stress and ferroptosis. eLife. 2014;3:e02523.

36. Sehm T, et al. Sulfasalazine impacts on ferroptotic cell death and alleviates the tumor microenvironment and glioma-induced brain edema. Oncotarget. 2016;7:36021-33

37. Lachaier $E$, et al. Sorafenib induces ferroptosis in human cancer cell lines originating from different solid tumors. Anticancer Res. 2014;34:6417-22.

38. Stockwell BR, Jiang X. A physiological function for Ferroptosis in tumor suppression by the immune system. Cell Metab. 2019:30:14-5.

39. Yang WS, Stockwell BR. Synthetic lethal screening identifies compounds activating iron-dependent, nonapoptotic cell death in oncogenic-RASharboring cancer cells. Chem Biol. 2008;15:234-45.

40. Weïwer $\mathrm{M}$, et al. Development of small-molecule probes that selectively kill cells induced to express mutant RAS. Bioorg Med Chem Lett. 2012;22:1822-

41. Yang WS, et al. Identification of simple compounds with microtubulebinding activity that inhibit Cancer cell growth with high potency. ACS Med Chem Lett. 2012;3:35-8. 
42. Yang WS, et al. Regulation of ferroptotic cancer cell death by GPX4. Cell. 2014;156:317-31.

43. Shimada K, et al. Global survey of cell death mechanisms reveals metabolic regulation of ferroptosis. Nat Chem Biol. 2016;12:497-503.

44. Woo $\mathrm{JH}$, et al. Elucidating compound mechanism of action by network perturbation analysis. Cell. 2015;162:441-51.

45. Jelinek A, et al. Mitochondrial rescue prevents glutathione peroxidasedependent ferroptosis. Free Radic Biol Med. 2018;117:45-57.

46. Friedmann Angeli JP, et al. Inactivation of the ferroptosis regulator Gpx4 triggers acute renal failure in mice. Nat Cell Biol. 2014;16:1180-91.

47. Stamenkovic A, Pierce GN, Ravandi A. Phospholipid oxidation products in ferroptotic myocardial cell death. Am J Physiol Heart Circ Physiol. 2019;317: H156-h163.

48. Hassannia B, et al. Nano-targeted induction of dual ferroptotic mechanisms eradicates high-risk neuroblastoma. J Clin Invest. 2018;128:3341-55.

49. Li Q, et al. Inhibition of neuronal ferroptosis protects hemorrhagic brain. JCl insight. 2017;2:e90777.

50. Fang S, Yu X, Ding H, Han J, Feng J. Effects of intracellular iron overload on cell death and identification of potent cell death inhibitors. Biochem Biophys Res Commun. 2018:503:297-303.

51. Eling N, Reuter L, Hazin J, Hamacher-Brady A, Brady NR. Identification of artesunate as a specific activator of ferroptosis in pancreatic cancer cells. Oncoscience. 2015;2:517-32.

52. Kim SE, et al. Ultrasmall nanoparticles induce ferroptosis in nutrientdeprived cancer cells and suppress tumour growth. Nat Nanotechnol. 2016; 11:977-85.

53. Phillips E, et al. Clinical translation of an ultrasmall inorganic optical-PET imaging nanoparticle probe. Science translational medicine. 2014;6: 260ra149.

54. Moss LR, Mulik RS, Van Treuren T, Kim SY, Corbin IR. Investigation into the distinct subcellular effects of docosahexaenoic acid loaded low-density lipoprotein nanoparticles in normal and malignant murine liver cells. Biochim Biophys Acta. 2016;1860:2363-76.

55. Ou W, et al. Low-density lipoprotein docosahexaenoic acid nanoparticles induce ferroptotic cell death in hepatocellular carcinoma. Free Radic Biol Med. 2017:112:597-607.

56. Liu T, et al. Ferrous-supply-regeneration Nanoengineering for Cancer-cellspecific Ferroptosis in combination with imaging-guided photodynamic therapy. ACS Nano. 2018;12:12181-92.

57. Zheng DW, et al. Switching apoptosis to Ferroptosis: metal-organic network for high-efficiency anticancer therapy. Nano Lett. 2017;17:284-91.

58. Bao W, et al. Nanolongan with multiple on-demand conversions for Ferroptosis-apoptosis combined anticancer therapy. ACS Nano. 2019;13: 260-73.

59. Huo M, Wang L, Wang Y, Chen Y, Shi J. Nanocatalytic tumor therapy by single-atom catalysts. ACS Nano. 2019;13:2643-53.

60. Westberg $\mathrm{M}$, et al. Exerting better control and specificity with singlet oxygen experiments in live mammalian cells. Methods (San Diego, Calif.). 2016;109:81-91.

61. Devos D, et al. A ferroptosis-based panel of prognostic biomarkers for amyotrophic lateral sclerosis. Sci Rep. 2019;9:2918.

62. Kauffman ME, et al. MitoSOX-Based Flow Cytometry for Detecting Mitochondrial ROS. Reactive oxygen species (Apex, N.C.). 2016;2:361-70.

63. Brown CW, et al. Prominin2 Drives Ferroptosis Resistance by Stimulating Iron Export. Developmental cell. 2019;51:575-86.e574.

64. Belavgeni A, Bornstein SR, Linkermann A. Prominin-2 suppresses Ferroptosis sensitivity. Dev Cell. 2019;51:548-9.

65. Mukaide T, et al. Histological detection of catalytic ferrous iron with the selective turn-on fluorescent probe RhoNox-1 in a Fenton reaction-based rat renal carcinogenesis model. Free Radic Res. 2014;48:990-5.

66. Chereddy NR, et al. A novel FRET 'off-on' fluorescent probe for the selective detection of $\mathrm{Fe}^{3+}, \mathrm{Al}^{3+}$ and $\mathrm{Cr}^{3+}$ ions: its ultrafast energy transfer kinetics and application in live cell imaging. Biosens Bioelectron. 2015;68:749-56.

67. Wang N, Zeng GZ, Yin JL, Bian ZX. Artesunate activates the ATF4-CHOPCHAC1 pathway and affects ferroptosis in Burkitt's lymphoma. Biochem Biophys Res Commun. 2019;519:533-9.

68. Sun $X$, et al. Activation of the p62-Keap1-NRF2 pathway protects against ferroptosis in hepatocellular carcinoma cells. Hepatology. 2016;63:173-84.

69. Knudsen ES, Knudsen KE. Tailoring to RB: tumour suppressor status and therapeutic response. Nat Rev Cancer. 2008;8:714-24.
70. Louandre $\mathrm{C}$, et al. The retinoblastoma ( $\mathrm{Rb})$ protein regulates ferroptosis induced by sorafenib in human hepatocellular carcinoma cells. Cancer Lett. 2015;356:971-7.

71. Zhang $X$, et al. Ferroptosis is governed by differential regulation of transcription in liver cancer. Redox Biol. 2019;24:101211.

72. Lo M, Ling V, Wang YZ, Gout PW. The xc- cystine/glutamate antiporter: a mediator of pancreatic cancer growth with a role in drug resistance. $\mathrm{Br} J$ Cancer. 2008;99:464-72.

73. Kerins MJ, Milligan J, Wohlschlegel JA, Ooi A. Fumarate hydratase inactivation in hereditary leiomyomatosis and renal cell cancer is synthetic lethal with ferroptosis induction. Cancer Sci. 2018;109:2757-66.

74. Yang WH, et al. A TAZ-ANGPTL4-NOX2 Axis regulates Ferroptotic cell death and Chemoresistance in epithelial ovarian Cancer. Mol Cancer Res. 2020;18: 79-90.

75. Tesfay $L$, et al. Stearoyl-CoA Desaturase 1 protects ovarian Cancer cells from Ferroptotic cell death. Cancer Res. 2019;79:5355-66.

76. Zhu S, et al. HSPA5 regulates Ferroptotic cell death in Cancer cells. Cancer Res. 2017;77:2064-77.

77. Qi W, et al. LncRNA GABPB1-AS1 and GABPB1 regulate oxidative stress during erastin-induced ferroptosis in HepG2 hepatocellular carcinoma cells. Sci Rep. 2019:9:16185.

78. Wang K, et al. Role of GRP78 inhibiting artesunate-induced ferroptosis in KRAS mutant pancreatic cancer cells. Drug Design Dev Ther. 2019;13:213544.

79. Harbeck N, Gnant M. Breast cancer. Lancet. 2017:389:1134-50

80. Pizzamiglio S, De Bortoli M, Taverna E, Signore M, Veneroni S, Cho WC, Orlandi R, Verderio P, Bongarzone I. Expression of Iron-Related Proteins Differentiate Non-Cancerous and Cancerous Breast Tumors. Int J Mol Sci. 2017;18(2):410.

81. Yu H, et al. Sulfasalazine-induced ferroptosis in breast cancer cells is reduced by the inhibitory effect of estrogen receptor on the transferrin receptor. Oncol Rep. 2019;42:826-38.

82. Doll S, et al. ACSL4 dictates ferroptosis sensitivity by shaping cellular lipid composition. Nat Chem Biol. 2017;13:91-8.

83. Mao C, et al. A G3BP1-interacting IncRNA promotes Ferroptosis and apoptosis in Cancer via nuclear sequestration of p53. Cancer Res. 2018;78: 3484-96.

84. Lin Y-S, et al. Danshen improves survival of patients with breast Cancer and Dihydroisotanshinone I induces Ferroptosis and apoptosis of breast Cancer cells. Front Pharmacol. 2019:10:1226.

85. Calderwood SK, Gong J. Heat shock proteins promote Cancer: It's a protection racket. Trends Biochem Sci. 2016;41:311-23.

86. Li S, Young KH, Medeiros L. Diffuse large B-cell lymphoma. Pathology. 2018, 50:74-87.

87. Kinowaki Y, et al. Glutathione peroxidase 4 overexpression inhibits ROSinduced cell death in diffuse large B-cell lymphoma. Lab Invest. 2018;98: $609-19$.

88. Chu B, et al. ALOX12 is required for p53-mediated tumour suppression through a distinct ferroptosis pathway. Nat Cell Biol. 2019;21:579-91.

89. Jemal A, et al. Global cancer statistics. CA Cancer J Clin. 2011;61:69-90.

90. Nie J, Lin B, Zhou M, Wu L, Zheng T. Role of ferroptosis in hepatocellular carcinoma. J Cancer Res Clin Oncol. 2018;144:2329-37.

91. Sun $X$, et al. Metallothionein-1G facilitates sorafenib resistance through inhibition of ferroptosis. Hepatology. 2016;64:488-500.

92. Bai T, et al. Haloperidol, a sigma receptor 1 antagonist, promotes ferroptosis in hepatocellular carcinoma cells. Biochem Biophys Res Commun. 2017;491: 919-25.

93. Yuan H, Li X, Zhang X, Kang R, Tang D. CISD1 inhibits ferroptosis by protection against mitochondrial lipid peroxidation. Biochem Biophys Res Commun. 2016;478:838-44

94. Tang S, Xiao X. Ferroptosis and kidney diseases. Int Urol Nephrol. 2019. https://doi.org/10.1007/s11255-11019-02335-11257.

95. Yang W-H, et al. The Hippo Pathway Effector TAZ Regulates Ferroptosis in Renal Cell Carcinoma. Cell Rep. 2019;28:2501-8.e2504.

96. Barnett R. Ovarian cancer. Lancet. 2016:387:1265.

97. Basuli D, et al. Iron addiction: a novel therapeutic target in ovarian cancer. Oncogene. 2017:36:4089-99.

98. Zhou HH, et al. Erastin reverses ABCB1-mediated Docetaxel resistance in ovarian Cancer. Front Oncol. 2019:9:1398. 
99. Greenshields AL, Shepherd TG, Hoskin DW. Contribution of reactive oxygen species to ovarian cancer cell growth arrest and killing by the anti-malarial drug artesunate. Mol Carcinog. 2017;56:75-93.

100. Carbone M, Melino G. Stearoyl CoA Desaturase regulates Ferroptosis in ovarian Cancer offering new therapeutic perspectives. Cancer Res. 2019;79: 5149-50.

101. Kamisawa T, Wood LD, Itoi T, Takaori K. Pancreatic cancer. Lancet. 2016;388: 73-85.

102. Badgley MA, et al. Cysteine depletion induces pancreatic tumor ferroptosis in mice. Science. 2020;368:85-9.

103. Yamaguchi Y, Kasukabe T, Kumakura S. Piperlongumine rapidly induces the death of human pancreatic cancer cells mainly through the induction of ferroptosis. Int J Oncol. 2018;52:1011-22.

104. Ma S, Henson ES, Chen Y, Gibson SB. Ferroptosis is induced following siramesine and lapatinib treatment of breast cancer cells. Cell Death Dis. 2016;7:e2307.

105. Wang $\mathrm{CK}$, et al. CHAC2 is essential for self-renewal and glutathione maintenance in human embryonic stem cells. Free Radic Biol Med. 2017 113:439-51.

106. Chen M-S, et al. CHAC1 degradation of glutathione enhances cystinestarvation-induced necroptosis and ferroptosis in human triple negative breast cancer cells via the GCN2-elF2a-ATF4 pathway. Oncotarget. 2017;8: 114588-602.

107. Shin D, Kim EH, Lee J, Roh J-L. Nrf2 inhibition reverses resistance to GPX4 inhibitor-induced ferroptosis in head and neck cancer. Free Radic Biol Med. 2018;129:454-62.

108. Roh J-L, Kim EH, Jang H, Shin D. Nrf2 inhibition reverses the resistance of cisplatin-resistant head and neck cancer cells to artesunate-induced ferroptosis. Redox Biol. 2017;11:254-62.

109. Xie Y, et al. The tumor suppressor p53 limits Ferroptosis by blocking DPP4 activity. Cell Rep. 2017;20:1692-704.

110. Luo M, et al. miR-137 regulates ferroptosis by targeting glutamine transporter SLC1A5 in melanoma. Cell Death Differ. 2018;25:1457-72.

111. Zhang K, et al. miR-9 regulates ferroptosis by targeting glutamic-oxaloacetic transaminase GOT1 in melanoma. Mol Carcinog. 2018;57:1566-76.

112. Ishimoto T, et al. CD44 variant regulates redox status in cancer cells by stabilizing the $\mathrm{xCT}$ subunit of system $\mathrm{xC}(-)$ and thereby promotes tumor growth. Cancer Cell. 2011;19:387-400.

113. Yoshida GJ, Saya H. Therapeutic strategies targeting cancer stem cells. Cancer Sci. 2016;107:5-11.

\section{Publisher's Note}

Springer Nature remains neutral with regard to jurisdictional claims in published maps and institutional affiliations.

Ready to submit your research? Choose BMC and benefit from:

- fast, convenient online submission

- thorough peer review by experienced researchers in your field

- rapid publication on acceptance

- support for research data, including large and complex data types

- gold Open Access which fosters wider collaboration and increased citations

- maximum visibility for your research: over $100 \mathrm{M}$ website views per year

At $\mathrm{BMC}$, research is always in progress.

Learn more biomedcentral.com/submissions 